\title{
SITUAÇÕES DE VULNERABILIDADE NA PECUÁRIA DE CORTE NO RIO GRANDE DO SUL: ENTRE POLÍTICAS PÚBLICAS E QUESTÕES RURAIS ATUAIS
}

\author{
Alessandra Matte* \\ Paulo Dabdab Waquil**
}

\begin{abstract}
Resumo
O objetivo do artigo foi compreender o grau de vulnerabilidade sobre diferentes questões rurais vinculadas à ação do Estado em relação aos meios de vida de pecuaristas de corte do sul do Rio Grande do Sul, analisando como esses produtores criam estratégias de enfrentamento ou de adaptação aos efeitos das situações de vulnerabilidade a que estão expostos. A abordagem dos meios de vida é utilizada como instrumental analítico para estudar situações de vulnerabilidade. Os dados empíricos provêm da realização de 60 entrevistas com pecuaristas de corte dos municípios de Bagé, Dom Pedrito, Pinheiro Machado e Piratini, ao sul do Rio Grande do Sul. Os resultados apontam que entre os setes fatores de vulnerabilidade analisados, a presença do Estado, por meio da atuação da prefeitura municipal e de agências de extensão e pesquisa, é importante ativo para reação às situações de vulnerabilidade quando acessível ao produtor rural. Os pecuaristas procuram em organizações locais, como sindicatos e associações de produtores, mecanismos para reagir à vulnerabilidade, assim como a mobilização de ativos internos à propriedade rural, quando da fragilidade do atendimento de serviços que deveriam ser oferecidos por políticas públicas. A superação dos desafios políticos envolve a constituição de sistemas de governança mais próximos às diferentes realidades rurais, facilitada quando compreendidas as situações de vulnerabilidade que podem ser geradas pela presença ou ausência do Estado.
\end{abstract}

Palavras-chave: Meios de vida. Pecuária de corte. Políticas públicas. Vulnerabilidade.

\footnotetext{
* Mestre e Doutora em Desenvolvimento Rural, pelo Programa de Pós-Graduação em Desenvolvimento Rural (PGDR), da Universidade Federal do Rio Grande do Sul (UFRGS). Pós-Doutoranda em Desenvolvimento Rural (DocFix CAPES/FAPERGS). E-mail: alessandramatte@yahoo.com.br

** Doutor em Economia Agrícola (University of Wisconsin, EUA).Professor dos Programas de Pós-Graduação em Desenvolvimento Rural (PGDR) e Agronegócios (CEPAN) da Universidade Federal do Rio Grande do Sul (UFRGS). E-mail: waquil@ufrgs.br
} 


\section{Introdução}

A atividade pecuária no sul do Rio Grande do Sul é resultado principalmente da conjunção de fatores políticos e naturais. A distribuição direcionada de áreas de terra e o incentivo à criação pecuária foram relevantes mecanismos de ação do Estado para delimitação territorial, facilitada pela presença das pastagens naturais do bioma Pampa, que favoreciam o desenvolvimento da atividade pecuária. É a partir desse contexto que este artigo aborda as diferentes formas como o Estado tem se mostrado presente junto à pecuária de corte, tomando por universo empírico o sul do Rio Grande do Sul. Desse modo, o objetivo deste artigo é compreender o grau de vulnerabilidade sobre diferentes questões rurais vinculadas à ação do Estado em relação aos meios de vida de pecuaristas de corte do sul do Rio Grande do Sul, analisando como esses produtores criam estratégias de enfrentamento ou de adaptação aos efeitos das situações de vulnerabilidade a que estão expostos.

Cabe destacar que o sul do Rio Grande do Sul, comumente conhecido pela tradição na criação pecuária, vem passando por um conjunto de transformações que apontam para consequências como a elevação do preço da terra, redução de áreas destinadas à pecuária sobre campos naturais, pressão à modernização da atividade, supressão do bioma Pampa e perda da biodiversidade, consequências estas potencialmente capazes de gerar diferentes situações de vulnerabilidade à atividade pecuária (MATTE, 2013; MATTE; WAQUIL, 2016). Preocupada em entender essas mudanças, à época pouco ou nada estudada, a pesquisadora Gabriela Litre (2010) desenvolveu sua tese por meio da investigação das percepções sobre vulnerabilidade e das estratégias de adaptação de pecuaristas de corte familiares do bioma Pampa da Argentina, do Uruguai e do Brasil. A autora constata que a atividade pecuária, por si só, é uma forma de reação às situações de vulnerabilidade, uma vez que os pecuaristas enxergam a atividade como um modo de vida e atrelam sua identidade à natureza.

Apesar dos avanços da agenda de pesquisa que tem tratado sobre o tema da vulnerabilidade, essa discussão - em que são prementes estudos que possam revelar como a vulnerabilidade opera em diferentes contextos rurais - está longe de ser esgotada. No conjunto de sua trajetória em estudos sobre situações de vulnerabilidade, Robert Chambers (2006) mostra quão variado é esse universo de pobreza para o qual buscamos explicações simples e soluções únicas. Para o autor, a vulnerabilidade é uma situação diretamente relacionada à exposição a contingências e às dificuldades de se lidar com elas, o que redunda em situações de mudanças e também incertezas (CHAMBERS, 2006). A contribuição desse debate está centrada em compreender como aqueles que passam por situações de vulnerabilidade 
reagem a elas, partindo do pressuposto de que o conjunto de estudos, para distintas formas de produção e realidades, pode oferecer uma ideia de como o Estado pode vir a atuar de maneira mais direta e eficiente com essas populações, de modo a auxiliá-las a superar essa situação. Conforme reconhecido por Chambers, os resultados desses estudos podem consistir em um caminho mais seguro para melhor compreensão e para ação que se ajustará de forma mais adequada e servirá à diversidade de condições e de pessoas, e às suas prioridades e necessidades.

Nesse cenário, a abordagem dos meios de vida (livelihoods) tem sido o principal instrumento para análise de situações de vulnerabilidade, uma vez que permite entender as diferenças em mesmos contextos rurais. Frank Ellis (2000), principal precursor dessa abordagem, apresenta contribuições para o debate dos ativos e das atividades das famílias rurais. Seu principal estudo acontece na Uganda, uma vez que sua agenda de pesquisas gira em torno da busca pela redução da pobreza em países de baixa renda. Em vista da facilidade de operacionalização, especialmente para análise comparativa, essa abordagem é comumente encontrada na linguagem de relatórios da Organização das Nações Unidas para Alimentação e Agricultura (FAO) e do Programa das Nações Unidas para o Desenvolvimento (PNUD), evidenciando a importância de adotar essa linguagem em estudos para a realidade brasileira.

Assim, entender situações de vulnerabilidade que podem ser geradas pela presença ou ausência do Estado por meio de diferentes políticas públicas, adotando como ferramenta de análise o uso da abordagem dos meios de vida, para a realidade da pecuária de corte no Rio Grande do Sul, mostra-se como importante contribuição para o contexto dos estudos rurais, especialmente considerando a relevância dessa atividade produtiva. Assim, este texto está estruturado em quatro partes, para além dessa introdução. A primeira contempla elementos sobre avanços na compreensão no conceito de situações de vulnerabilidade e no uso da abordagem dos meios de vida. A terceira parte é dedicada à apresentação dos instrumentos que descrevem o método de estudo. Os resultados são apresentados, analisados e discutidos na quarta parte do texto. Por fim, considerações finais são apresentadas a partir das análises desenvolvidas neste texto.

\section{Vulnerabilidade e meios devida:entre estratégias de enfrentamento e de adaptação}

A utilização do conceito de vulnerabilidade varia de significado em seus domínios de investigação, podendo ser encontrado em distintas áreas do conhecimento, como na Economia, na Ecologia, na Sociologia, nas Engenharias, na saúde, entre outras. Entre as principais referências no uso do termo, estão pesquisas desenvolvidas por Neil Adger $(2003,2006)$ 
e John Morton (2007), com foco especialmente sobre vulnerabilidade a mudanças climáticas. Olhando situações de vulnerabilidade social e demográficas, Eduardo Marandola Jr. e Daniel Hogan (2005, 2006) apresentam contribuições a partir de outra perspectiva. Esses exemplos permitem ilustrar a multifuncionalidade no uso do termo, uma vez que, no dinamismo da vulnerabilidade, há uma grande variedade de fatores e que esses fatores estão em constante mudança.

Por isso, a abordagem da vulnerabilidade leva em consideração o estresse provocado pelas mudanças ambientais e sociais, potencializado pela ausência de capacidade de adaptação, que pode ser representada pela própria ausência do Estado (AGDER, 2006; ADGER et al., 2004). Segundo Morton (2007), a vulnerabilidade pode proceder de várias tendências socioeconômicas, demográficas e políticas, limitando a capacidade de adaptação a mudanças. Já Adger (2006, p. 270) afirma que a vulnerabilidade é o "[...] estado de suscetibilidade a danos, causados por exposição ao estresse, associado com mudança ambiental e social e da ausência de capacidade de adaptação". Morton (2007) e Adger (2006) propõem pensar a vulnerabilidade em uma perspectiva que envolve a falta ou dificuldade de resiliência. Assim como esses autores, os estudos demográficos da Comissão Econômica para América Latina e Caribe (CEPAL, 2002) definem a vulnerabilidade como uma incapacidade de enfrentar os riscos ou como impossibilidade de manejar ativos para proteger-se diante das incertezas, podendo ser fruto também de uma omissão do Estado. Ao abordar essa forma de vulnerabilidade no Brasil e na América Latina, Nobre (2008) considera que ambos os lugares podem apresentar vulnerabilidade a fatores socioeconômicos e ambientais, com relativa significância as mudanças climáticas, especialmente sobre a agricultura.

Desde uma perspectiva multidimensional do estudo da vulnerabilidade, considera-se que os parâmetros promotores de vulnerabilidade se delineiam a partir de uma conjunção de fatores, podendo estar relacionados a um conjunto de acontecimentos que tornam um grupo ou indivíduo vulnerável, e não somente ligado a uma única variável (vulnerável versus não vulnerável). Conforme Cutter (1996), a riqueza da aplicação da perspectiva multidimensional da vulnerabilidade ocorre pela diversidade de formas em que o tema vem sendo abordado, englobando a variedade encontrada na política, nos hábitos culturais e sociais, no ambiente físico, além das implicações práticas e metodológicas para esses estudos aplicadas em cada local, que vão orientar o levantamento dos fatores de vulnerabilidade. Portanto, não basta apenas compreender o que é vulnerabilidade, é necessário verificar quais os motivos para o grupo ou indivíduo não conseguir criar estratégias para enfrentar ou se adaptar, o que pode estar diretamente relacionado às capacidades individuais e 
aos ativos de que dispõem para enfrentar essa situação. Segundo Janssen e Ostrom (2006), os desafios para a pesquisa da vulnerabilidade são desenvolver medidas robustas e críveis que incorporem diversos métodos que incluam a percepção de risco e vulnerabilidade, procurando contribuir com os mecanismos de governança que deveriam intermediar os fatores de vulnerabilidade, promovendo ações de adaptação.

Nesse cenário, Chambers (2006) e Ellis (2000) assumem que a vulnerabilidade está relacionada a uma falha dos direitos e à escassez de recursos. Assim, a presença de situações de vulnerabilidade representa uma privação ou ausência de liberdade que os indivíduos têm para escolher como querem viver, limitando sua capacidade de executar certas funções básicas, enfrentando situações de estresse e choques, com o objetivo de alcançar o meio de vida almejado. Portanto, em circunstâncias em que o indivíduo ou a família tiverem seus meios de vida ameaçados (por incertezas, riscos ou mudanças), sua liberdade de exercer suas capacidades estará comprometida, limitando suas escolhas e possibilidades de reação (ELLIS, 2000).

A abordagem dos "meios de vida" (livelihoods) proposta por Frank Ellis (2000) considera que as famílias desenvolvem suas estratégias de reprodução social estabelecendo ligação entre os ativos e as atividades que o grupo familiar possui para sobreviver. Segundo o autor, "[...] um meio de vida compreende os bens (natural, físicos, humanos, financeiro e capital social), as atividades e o acesso a estas (mediados pelas instituições e relações sociais) que juntos determinam a vida adquirida pelo indivíduo ou pelo grupo familiar" (ELLIS, 2000, p. 10). Portanto, o meio que o indivíduo possui para viver envolve os ativos de que ele dispõe, suas atividades e as formas de acesso e uso para sobreviver que determinam o seu modo de viver.

Os meios de vida são compostos por um conjunto de cinco capitais (capital natural, físico, humano, financeiro e social) constituídos por diversos ativos, em que a condição em que se encontram influencia a forma como serão acessados e mobilizados, tendo como principal propósito a busca pelo sustento do estabelecimento e autonomia da família (SCOONES, 2009; ELLIS, 2000). O capital natural refere-se à base de recursos naturais relacionada à qualidade e quantidade de bens como terra, água, solo, entre outros -, e oferece produtos úteis à sobrevivência da população humana. O capital físico pode ser exemplificado por ferramentas, maquinários e insumos disponíveis, ou seja, pelos bens trazidos à existência pelo processo de produção econômica. $\mathrm{O}$ capital humano está relacionado ao trabalho doméstico disponível, às atribuições dos indivíduos, como nível de escolaridade, conhecimento, habilidades e o próprio estado de saúde. No que diz respeito ao capital financeiro, refere-se ao estoque de dinheiro, poupança e crédito, os quais podem ser acessados a fim de adquirir bens 
tanto de produção como de consumo. Por fim, o capital social corresponde a redes de reciprocidade, confiança e associações das quais as pessoas participam e a partir das quais podem derivar apoio que contribui para seu sustento (ELLIS, 2000).

Portanto, os indivíduos e as famílias possuem diferentes formas de acesso aos distintos capitais, atribuindo heterogeneidade em suas estratégias de enfrentamento e adaptação às diversas situações de vulnerabilidade (CHAMBERS, 2006; ADGER et al., 2008). Assim, entender a diversidade dos grupos familiares torna-se fundamental na medida em que possibilita compreender as opções que estão à disposição dos indivíduos, bem como as estratégias que estes adotam frente às situações de vulnerabilidade. $\mathrm{Na}$ perspectiva de Ellis (2000), a vulnerabilidade representa uma mudança em que o indivíduo, a família ou a comunidade pode estar vivenciando, tratando-se de uma mudança particular na sustentabilidade dos meios de vida. Diante de uma situação de vulnerabilidade, os indivíduos podem reagir de duas maneiras: enfrentando ou adaptando-se.

As estratégias de enfrentamento têm como objetivo a sobrevivência, buscando criar alternativas para superar essa situação em face das variações indesejáveis (ELLIS, 1999, 2000; CHAMBERS; CONWAY, 1992; ADGER, 2003). Consistem em medidas para moderar ou reduzir os riscos ou mesmo seus impactos, ainda que temporariamente. As estratégias de adaptação, por sua vez, envolvem a capacidade dos meios de vida "evoluírem", a fim de acomodar as situações de riscos ou mudança, ampliando a gama de variabilidade com que podem lidar com as situações de vulnerabilidade (ADGER, 2006). Como essas estratégias de adaptação são manifestações desenvolvidas para assegurar a sobrevivência do grupo familiar em longo prazo, as adaptações de sucesso tornam as famílias menos propensas às crises ao longo do tempo, aperfeiçoando sua capacidade de resistir às mudanças e aos choques (CHAMBERS, 2006; KIRSCH et al., 2016).

Logo, compreender como os atores e as famílias reagem às diferentes situações de vulnerabilidade a que estão expostas relaciona-se diretamente com as privações que os atores sociais devem superar. Nesse cenário, o Estado, por meio da ação de políticas públicas ou por meio da atuação de organizações que têm atuação direta com o produtor rural, podem vir a ser instrumento para reagir a situações de vulnerabilidade ou então ser, por si só, o promotor de vulnerabilidade. Com base nessa reflexão, na próxima seção é apresentado o método que orientou o estudo para entender a relação da pecuária de corte com diferentes questões rurais vinculadas ao papel do Estado. Ressalta-se que conhecer as estratégias adotadas em situações de mudanças é um passo fundamental para fazer possíveis previsões quanto às reações a serem adotadas pelos indivíduos e famílias. 


\section{Método de Pesquisa}

Este estudo tem seu foco de análise nas famílias de pecuaristas de corte dos municípios de Bagé, Dom Pedrito, Piratini e Pinheiro Machado, situados ao sul do Rio Grande do Sul, região com predominância da atividade pecuária. Ao abordar a atividade pecuária é necessário compreender que não se trata de uma atividade com estilos de produção homogêneos, visto que há diversidade nos meios de vida dos pecuaristas bem como nas formas como estes desenvolvem suas atividades. Assim, não houve pretensão neste estudo de esgotar a diversidade de modos de produção da pecuária de corte, mas de atentar para a diversidade de formas com que esses pecuaristas vêm realizando a atividade e, desse modo, identificar as situações de vulnerabilidade e as estratégias de enfrentamento ou de adaptação adotadas.

Figura 1 - Localização dos municípios de Bagé, Dom Pedrito, Piratini e Pinheiro Machado no sul do Rio Grande do Sul

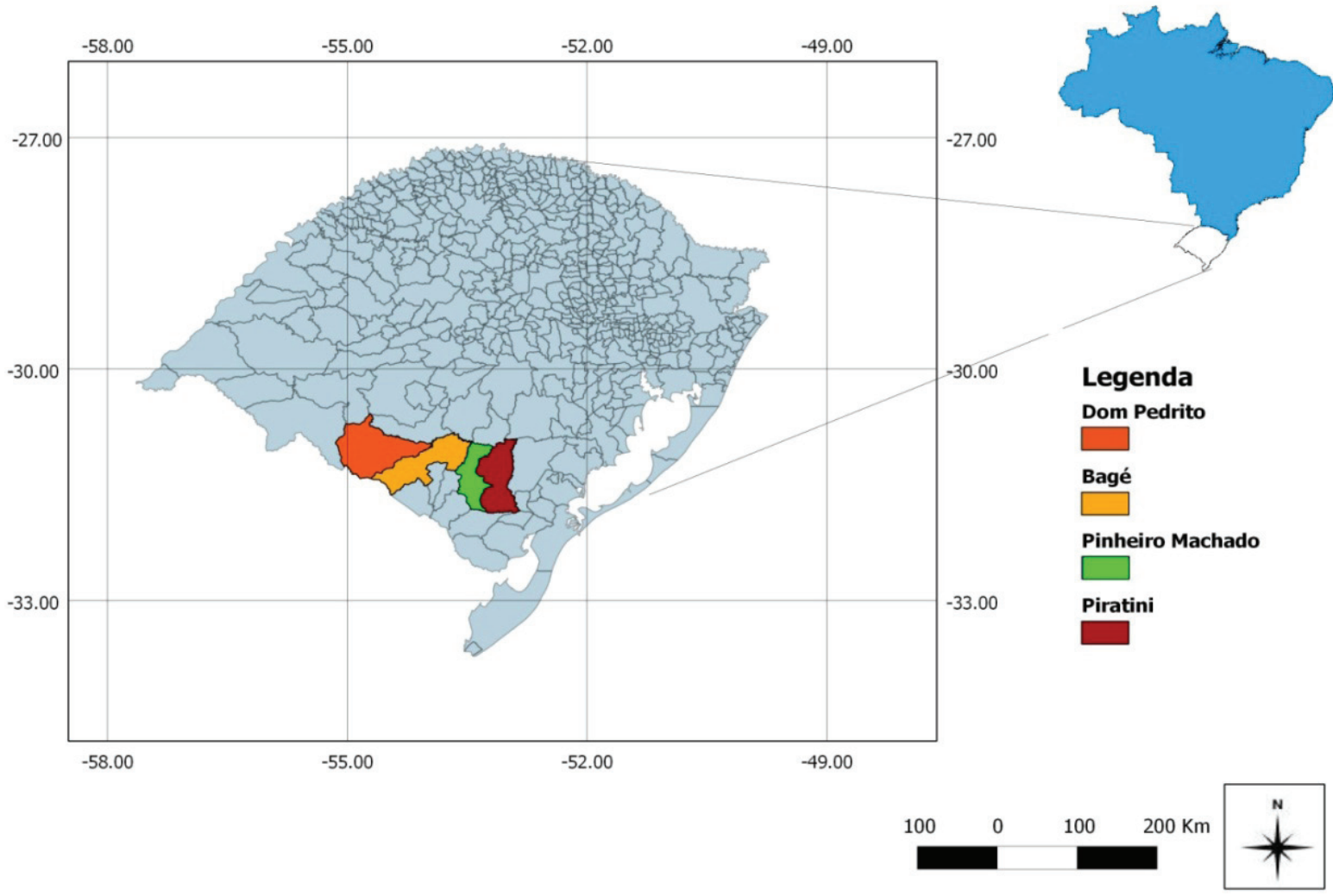

Fonte: Elaborada pelos autores (2018). 
Partindo da delimitação que considerou a presença da pecuária de corte no estabelecimento, a escolha dos entrevistados teve como principal objetivo abranger a diversidade de meios de vida dos pecuaristas de corte nos municípios investigados. O principal instrumento de coleta de informações foi entrevista face a face, realizada com 60 pecuaristas que desenvolvem a atividade pecuária. A entrevista é do tipo semiestruturada, a qual ocorre por meio de uma "conversação guiada", com o objetivo de obter informações detalhadas daquilo que está sendo investigado (RICHARDSON, 2009, p. 208).

A entrevista consistiu em um roteiro com questões abertas, fechadas e de múltipla escolha, de caráter qualitativo e quantitativo. Os dados quantitativos da pesquisa foram utilizados no tratamento das informações relativas aos meios de vida dos pecuaristas (ativos e atividades) e na identificação dos fatores de vulnerabilidade e seus graus de importância. Os dados qualitativos estão relacionados à compreensão da vulnerabilidade e das estratégias de enfrentamento e de adaptação adotadas pelos pecuaristas. As informações qualitativas foram transcritas e codificadas pela análise de conteúdo. Foram elencados, a partir de revisão de literatura sobre pecuária e de pesquisa exploratória, sete fatores de vulnerabilidade que possuem alguma relação com o Estado, analisados na próxima seção.

\section{Infraestrutura e fatores organizacionais na pecuária de corte: políticas públicas e ação do Estado}

As famílias entrevistadas nos quatro municípios desenvolvem a pecuária de corte por meio da criação de bovinos, ovinos ou caprinos, ocupando em média $87 \%$ da área total da propriedade. No que diz respeito ao tamanho da área dos estabelecimentos, há uma amplitude significativa no que se refere à área total, com propriedades de 5 a 3.320 hectares. Os animais são criados em sua totalidade (100\%) sobre pastagem nativa característica do bioma Pampa, a qual consiste no principal alimento utilizado para os animais em $87 \%$ dos estabelecimentos.

$\mathrm{O}$ conjunto de fatores analisados representam recursos e meios de que as famílias de pecuaristas de corte podem dispor para o enfrentamento ou a adaptação às diferentes situações de vulnerabilidade a que podem vir a ser expostos. $\mathrm{Na}$ mesma medida, a ausência desses recursos e serviços representa situação que inibe o exercício pleno de acesso aos meios de vida. Desse modo, as situações de vulnerabilidade aqui analisadas compreendem 
essencialmente sete fatores, os quais serão tratados individualmente ao longo do texto. O Gráfico 1 apresenta e ilustra os fatores de vulnerabilidade que compõem o estudo, com sua distribuição nos respectivos graus de importância.

Gráfico 1 - Fatores de vulnerabilidade e graus de importância de aspectos relacionados à infraestrutura e a fatores organizacionais

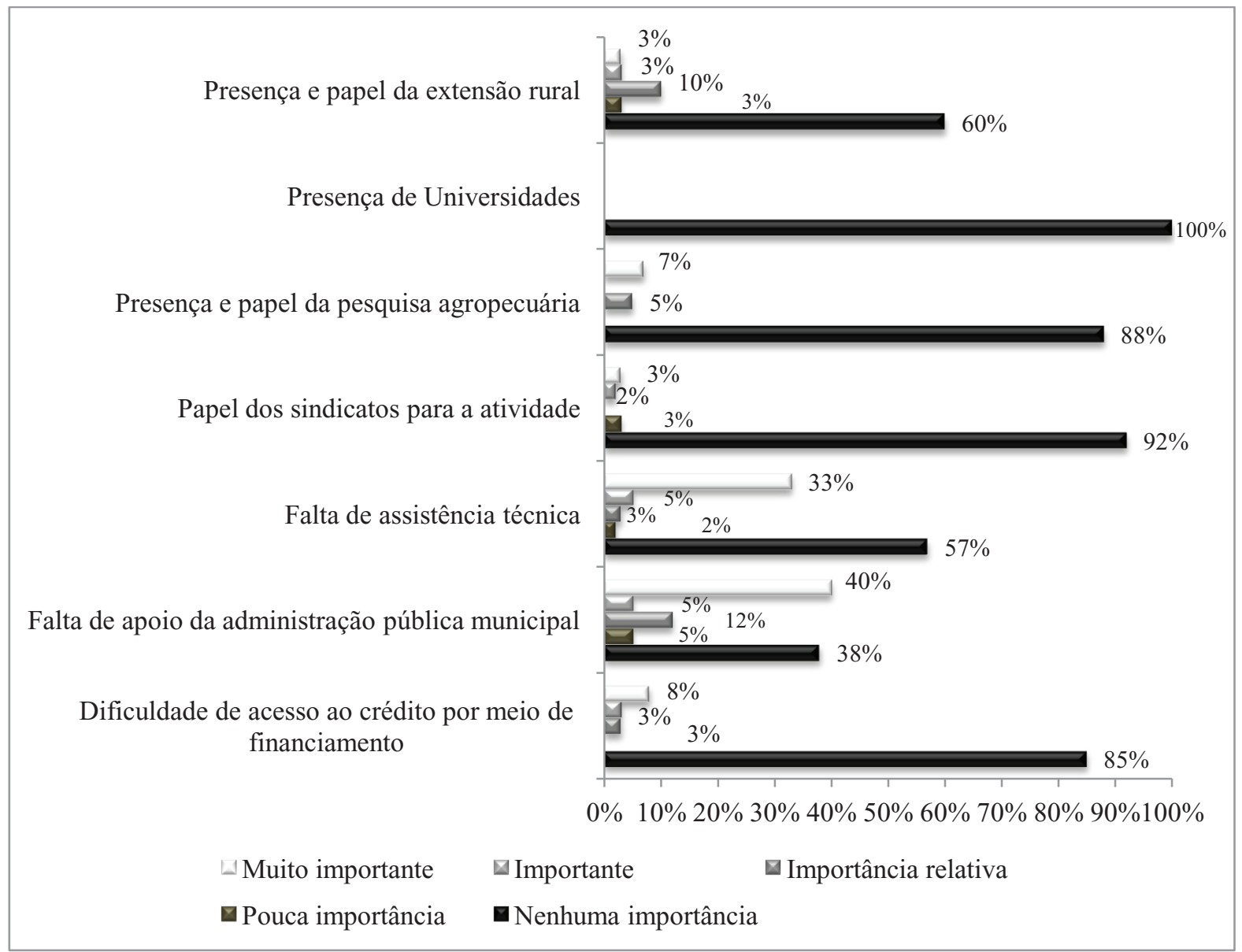

Dados da pesquisa de campo.

Fonte: Elaborado pelos autores (2018).

Naturalmente, a importância da pecuária para as famílias entrevistadas não está relacionada apenas com fatores econômicos, mas especialmente com questões tradicionais. Nesse sentido, estudos realizados por Cotrim (2003), Sandrini (2005) e Ribeiro (2016) conseguem demonstrar que a atividade pecuária representa muito mais que uma atividade com fins lucrativos. De maneira geral, ela está diretamente ligada às questões tradicionais e de satisfação pessoal, que carregam consigo valores morais e uma simbologia 
própria. Em estudo realizado por Andreatta, Waquil e Miguel (2016), os resultados apontam que a tradição é um dos principais motivos para os pecuaristas atuarem na atividade pecuária, representando $26,5 \%$ de um universo de 540 entrevistados em todo o estado do Rio Grande do Sul. Tendo isso em vista, para facilitar a análise e a discussão dos resultados, esta seção está subdividida com base nos sete fatores de vulnerabilidade analisados, na sequência ilustrada no gráfico.

\section{a) Dificuldade de acesso ao crédito por meio de financiamento}

O primeiro fator de vulnerabilidade refere-se à dificuldade de acesso ao crédito, representando uma vulnerabilidade para uma parcela muito pequena do total de entrevistados (15\%) e sendo considerado muito importante para apenas $8 \%$ deles. Esse fator não representa uma vulnerabilidade para a maioria dos pecuaristas, apresentando nenhuma importância para 85\% dos entrevistados. Esse elevado valor se justifica pelo fato de $62 \%$ dos entrevistados não acessarem qualquer forma de crédito rural.

Em sua maioria, os entrevistados têm aversão a acessar financiamento por considerarem o condicionamento ao pagamento da dívida uma situação de risco e incerteza, visto que a atividade pecuária não gera renda regular, mas por temporada de comercialização dos animais. E, entre aqueles que acessam, o destino do crédito divide-se em custeio para a pecuária de corte e para a lavoura. Observa-se, ainda, uma correlação média entre tamanho do estabelecimento e acesso ao crédito $(0,32)$, apontando que quanto menor a área de terra do estabelecimento, menor o acesso ao crédito. $\mathrm{O}$ mesmo é observado com relação à renda pecuária: quanto menor a renda auferida com a atividade pecuária, menor o acesso ao crédito $(0,38)$.

Situação similar foi também identificada entre pecuaristas familiares em estudo desenvolvido por Ribeiro (2016). O autor destaca que tal situação é representativa de um comportamento econômico que demonstra aversão ao risco. Os resultados dessa pesquisa revelam que a aversão ao risco não está presente somente entre os pecuaristas familiares, mas também nos demais estilos de pecuária, corroborando com os resultados de Andreatta, Waquil e Miguel (2016) sobre a realidade da pecuária de corte no estado do Rio Grande do Sul.

Assim, a opção de não acessar financiamentos representa uma escolha desses indivíduos e não uma falta ou carência de ativos, ou seja, eles estão realizando suas escolhas sem comprometer seus meios de vida ao optarem por não acessar o crédito. Para o pequeno grupo de pecuaristas para os quais este fator representa uma vulnerabilidade, os motivos envolvem as dificuldades para pagar o financiamento, enquanto para outros, está a dificuldade em acessar o crédito. 


\section{b) Falta de apoio da administração pública municipal}

A falta de apoio da administração pública municipal representa o segundo fator de vulnerabilidade, considerado assim um total de $62 \%$ dos entrevistados, distribuídos em todos os graus de importância: 5\% com pouca importância; 12\%, importância relativa; 5\%, importante, e 40\%, muito importante. A relevância do poder público municipal impacta de diferentes maneiras nos estabelecimentos rurais, como evidenciado por Adger (2006) em estudo sobre os impactos das mudanças climáticas. O autor reconhece que a vulnerabilidade pode resultar de falha do acesso à rede pública de serviços, uma vez que esta, por si só, pode representar um mecanismo de promoção de ação adaptativa e de resiliência. Apenas $38 \%$ dos entrevistados não apontam esse fator como uma vulnerabilidade, não lhe atribuindo nenhuma importância. A variação de percepções em torno desse fator deve-se às diferenças na administração pública realizada nos quatro municípios e suas ações no meio rural. Nesse caso, a ação municipal representa uma vulnerabilidade quando se espera dela o apoio no enfrentamento e adaptação às situações de vulnerabilidade (por exemplo: seca, falta de água), e principalmente o atendimento às necessidades básicas (por exemplo: educação, atendimento à saúde, manutenção das estradas etc.).

Para os entrevistados, o apoio da prefeitura é parcial. Para muitos, a organização nem sempre realiza suas obrigações, deixando a desejar em aspectos que variam entre os municípios investigados. Apesar do alto grau de importância atribuído ao papel da administração pública, os entrevistados demonstram estar adaptados à situação, uma vez que permanecem realizando suas atividades sem uma dependência direta das ações administrativas dos municípios. Assim, a ausência de apoio do município não impede esses pecuaristas de reproduzirem seus meios de vida, visto que já estão adaptados a realizar suas atividades sem, ou quase sem, apoio da participação da administração pública. No entanto, esses indivíduos reconhecem que poderia ser um importante ativo do meio de vida da família, se de fato a prefeitura cumprisse com o atendimento das condições básicas estabelecidas em lei.

Uma das vias importantes de enfrentamento à falta de apoio da administração pública ocorre mediante a mobilização coletiva por meio das associações de pecuaristas. Essa situação se reflete na expansão das capacidades humanas que ajudam direta ou indiretamente a enriquecer os meios de vida e tornar as privações mais raras e menos aflitivas. Assim, a associação de pecuaristas age representando e dando voz a um grupo de produtores, tornando possível a reivindicação de seus direitos e interesses, por exemplo, boas estradas, acesso à saúde e à educação. 
Em estudo sobre a vulnerabilidade climática entre agricultores do Mato Grosso, Kirsch (2015) reconhece que o funcionamento do quadro institucional cria a possibilidade de mobilização de uma diversidade maior de arranjos e formas de produção. Portanto, segundo o autor, tais reações influenciam na geração de situações que podem implicar uma fragilidade ou mesmo uma incapacidade de enfrentar ou de se adaptar às mudanças ambientais contemporâneas. Torna-se evidente que o protagonismo da prefeitura municipal, enquanto primeira instância de vínculo com o Estado, pode vir a oferecer importantes ativos para o enfrentamento a diferentes situações de vulnerabilidade, seja pelo atendimento a serviços básicos, seja pela constituição de programas e políticas locais adaptadas à realidade regional.

\section{c) Falta de assistência técnica}

Dando sequência, o terceiro fator de vulnerabilidade é representado pela falta de assistência técnica, percebido como uma vulnerabilidade para um total de $43 \%$ de entrevistados, com alto grau de importância para 33\% deles. Segundo Deponti, Scarton e Schneider (2012), no Rio Grande do Sul a orientação técnica do governo (federal, estadual e municipal) possui a maior representatividade, sendo responsável pela assistência em 31\% dos estabelecimentos, enquanto cooperativas e empresas integradoras são responsáveis pela orientação em 26\% e 24\% dos estabelecimentos, respectivamente. Segundo o Censo Agropecuário 2006 (IBGE, 2009), somente $7 \%$ dos estabelecimentos receberam orientação disponibilizada por empresas privadas, ao passo que a orientação técnica própria ou do próprio produtor foi utilizada em $10 \%$ dos estabelecimentos que receberam orientação técnica.

Assim, aqueles pecuaristas que estão adaptados realizam o pagamento pelo serviço de assistência técnica, enquanto outros pecuaristas que não dispõem de capital financeiro para pagar pelo serviço acabam enfrentando esse fator de vulnerabilidade, contando com a troca de experiência entre pecuaristas e com a colaboração de vizinhos e familiares com formação técnica ou superior. $\mathrm{O}$ pagamento pela assistência técnica é realizado com base na mobilização de recurso financeiro próprio, realizado por pecuaristas que dispõem desse capital. Essa estratégia é realizada com certa periodicidade para garantir a manutenção do rebanho, sendo o responsável técnico contratado pelo pecuarista.

As duas estratégias seguintes envolvem o fortalecimento do capital social no enfrentamento à necessidade de assistência técnica e a reciprocidade entre pecuaristas, que representa outra forma de enfrentar essa situação de vulnerabilidade gerada pela falta de acesso à assistência técnica. A associação 
de produtores é elemento fundamental na construção de estratégias de enfrentamento, servindo de ambiente de troca de informações e experiências, além da construção de estratégias de enfrentamento com base nos meios de vida de que dispõem. São igualmente importantes as ações coletivas e individuais no âmbito das comunidades, da família e de outros círculos não governamentais, mas que também agem para ajustar as incertezas, diminuindo assim a vulnerabilidade (SCOONES, 2009; RIBOT, 2009; HERTEL; ROSCH, 2010). A outra estratégia de enfrentamento conta com o capital humano disponível, por meio do conhecimento de algum membro da família com formação superior ou técnica.

Essa forma de utilização da assistência técnica (por meio da troca) é observada principalmente nos pequenos estabelecimentos, justificada por fatores como baixa renda e dificuldades na contratação de assistência técnica esporádica temporária. Essa ajuda mútua na forma de troca de serviço e favores não envolve pagamento monetário, mas sim valores de reciprocidade, não sendo classificada como contratação de mão de obra (SABOURIN,2009). A ajuda mútua presente nessas relações é denominada por Sabourin (2009) de reciprocidade, criando um valor ético, de relações que se redobram, entendida como a dinâmica de reprodução de prestações geradora de vínculo social. Por outro lado, a reciprocidade, enquanto relação social construída para suprir a carência ou falta de assistência técnica, não é evidente em propriedades de maior porte, as quais fazem uso de profissionais contratados.

\section{d) Papel dos sindicatos para a atividade}

O quarto fator de vulnerabilidade é o papel dos sindicados para a atividade pecuária. Esse fator não é considerado uma vulnerabilidade para $92 \%$ dos entrevistados, e, dentre aqueles que o consideram uma vulnerabilidade, tem pouca importância para $3 \%$, é importante para $2 \%$, e muito importante para 3\%. Podemos destacar que a baixa vulnerabilidade a esse fator tem relação direta com a participação dos sindicatos no enfrentamento e na adaptação de diferentes situações de vulnerabilidade. Cabe reconhecer que a principal entidade representativa reconhecida pelos pecuaristas é o Sindicato de Trabalhadores Rurais, ao qual estão vinculados 65\% dos entrevistados. Para esses indivíduos, a associação ao sindicato oferece benefícios no acesso à saúde da família, na vacinação dos animais, na aquisição de sementes para o cultivo de pastagens e, em alguns casos, na promoção de cursos e capacitações para os pecuaristas.

Nessa situação, as famílias estão obtendo os benefícios do capital social que compõem os seus meios de vida por meio do apoio do sindicato para a manutenção e reprodução da família. Esse fator representa uma 
vulnerabilidade quando as ações do sindicato não correspondem às necessidades dos pecuaristas, sendo observada essa percepção somente sobre o sindicato patronal. Não são identificadas estratégias de enfrentamento e adaptação, apenas reivindicações por parte dos entrevistados quanto à necessidade de melhorias nas formas de apoio dadas pelo sindicato.

Em estudo sobre vulnerabilidade e pobreza no território da Zona Sul no Rio Grande do Sul, Toigo e Conterato (2015) constatam que uma ação mais integrada entre o Estado, as instituições e as comunidades rurais possa ser um caminho que assegure uma coesão social mais sólida e efetiva enquanto estratégia para reação a situações de vulnerabilidade. Os resultados dos autores corroboram com os encontrados nesta pesquisa, visto que reforçam a importância de organizações locais como ativo a ser mobilizado do conjunto de capitais que compõem os meios de vida diante de situações que possam expor as famílias a riscos.

\section{e) Presença e papel da pesquisa agropecuária}

O quinto fator de vulnerabilidade refere-se ao papel da pesquisa agropecuária desenvolvida pela Empresa Brasileira de Pesquisa Agropecuária (Embrapa), que, assim como o fator anterior, é considerada com grau de nenhuma importância para $88 \%$ dos entrevistados. Isso se justifica pelo apoio oferecido por parte dessa instituição, representando um importante ativo nos meios de vida dos pecuaristas entrevistados. Para o pequeno grupo que considera a Embrapa como um fator de vulnerabilidade, a instituição passou a representar uma vulnerabilidade mediante sua ausência em apoio tecnológico; afinal, consistindo em um ativo nos meios de vida dos pecuaristas, seu afastamento significa a perda de um mecanismo para o enfrentamento ou adaptação às situações de vulnerabilidade, restringindo, assim, as oportunidades de escolha.

Entre aqueles que não consideram o papel da Embrapa como uma vulnerabilidade (88\%) estão dois grupos de pecuaristas: aqueles que nunca tiveram contanto com a instituição e suas ações - portanto, não sentem interferência desta nos meios de vida da família -, e aqueles que veem a Embrapa como importante ativo no enfrentamento e adaptação às situações de vulnerabilidade, considerando-a como parte dos meios de vida de suas famílias.

Assim, para o segundo grupo, a participação da Embrapa tem ocorrido, principalmente, por meio do desenvolvimento do Projeto Alto Camaquã, coordenado pela organização com sede em Bagé, em que conduzia ações de promoção do desenvolvimento endógeno da região compreendida pelo território Alto Camaquã, abarcando municípios de estudo, Bagé, Pinheiro Machado e Piratini (BORBA; TRINDADE, 2009; 
MATTE et al., 2016). O projeto envolve uma rede de atores sociais locais e regionais, como organizações de pesquisa, ensino, extensão, poder público municipal e estadual, sindicatos e associações de produtores, e abrange os municípios que compõem o chamado território Alto Camaquã. As ações do Projeto Alto Camaquã ocorrem juntamente com as associações de produtores por meio de pesquisas participativas. Devido a isso, a Embrapa é considerada pelos pecuaristas como uma aliada na construção de estratégias para enfrentar e se adaptar às diversas situações de vulnerabilidade.

Essa percepção por parte dos entrevistados se efetiva principalmente pelo papel da organização no apoio e valorização desses indivíduos, de suas famílias e da atividade pecuária. Constatou-se que a presença e ação da Embrapa têm fortalecido o capital social dos pecuaristas, uma vez que o trabalho coletivo tem permitido encontrar soluções para reagir às situações de vulnerabilidade a que os pecuaristas estão expostos, por exemplo, o desenho de estratégias de manejo do campo nativo que permitem um melhor uso desse recurso (campo) em períodos de seca e inverno. Como o Projeto Alto Camaquã tem diversificado o portfólio de ativos, contribui para ampliar oportunidades e a própria capacidade de escolha dos entrevistados.

Por outro lado, parte significativa dos entrevistados tem uma relação muito distante com a Embrapa. A organização não representa uma vulnerabilidade, mas também não é vista como um ativo, uma vez que não exerce qualquer influência sobre os meios de vida. Assim, os pecuaristas estão adaptados à ausência de apoio da organização.

\section{f) Presença de Universidades}

O papel de universidades é o sexto fator de vulnerabilidade, sendo o único a não representar qualquer grau de vulnerabilidade entre os entrevistados. A presença de universidades na região representa um importante ativo disponível. Sua importância é ressaltada enquanto um ativo na estratégia de enfrentamento e adaptação a dois fatores de vulnerabilidade: a ausência de sucessores e as dificuldades no acesso à educação (MATTE; WAQUIL, 2013, 2016). Para ambos os fatores, a universidade representa um importante ativo, permitindo aos indivíduos incluírem em suas escolhas individuais a possibilidade e a oportunidade de cursar o ensino superior sem sair ou se afastar muito do estabelecimento.

\section{g) Presença e papel da extensão rural}

Por fim, deve-se mencionar a extensão rural representada pela Empresa de Assistência Técnica e Extensão Rural do Governo do Rio Grande do Sul (Emater/RS), o último fator de vulnerabilidade estudado. A extensão rural da Emater é considerada uma vulnerabilidade para um 
total de $40 \%$ dos entrevistados, com grau de importância muito alto para $23 \%$ deles. Por outro lado, para $60 \%$ dos entrevistados não representa uma vulnerabilidade, o que significa que $\dot{\boldsymbol{e}}$ um importante ativo de apoio no enfrentamento e na adaptação às situações de vulnerabilidade. Então, a restrição no acesso ao serviço da Emater pode causar vulnerabilidade ao limitar os ativos disponíveis nos meios de vida para a maioria dos pecuaristas entrevistados.

Atualmente a Emater é a instituição de extensão rural que atua como veículo de muitas políticas públicas e de projetos governamentais, sendo sua incumbência aplicá-los, além de realizar a orientação e a assistência técnica aos produtores. Devido à multiplicidade de funções atribuídas à Emater, sua ação com os pecuaristas tem ficado comprometida.

Os discursos dos pecuaristas entrevistados, de modo geral, envolvem uma crítica à atual falta de acompanhamento. Muitos revelam que estão sem qualquer tipo de assistência técnica ou que precisam de procurar outra forma de solucionar essa carência, como já discutido anteriormente. Com base no Censo Agropecuário de 2006, Deponti, Scarton e Schneider (2012) apontam que $50 \%$ da população rural do Rio Grande do Sul não recebe algum tipo de orientação técnica, apesar de a Emater/RS estar presente em 99\% dos municípios gaúchos. Para os pecuaristas, a forma de tentar amenizar esse problema é buscar na própria organização algum tipo de ajuda quando realmente houver necessidade; caso contrário. a estratégia que adotam é dar continuidade à atividade da forma como se encontra. Somado a isso, os pecuaristas apontam que há um baixo número de funcionários na instituição, o que acaba comprometendo o atendimento e a orientação aos produtores.

\section{Considerações finais}

De maneira geral, os fatores analisados mostram um Estado presente entre pecuaristas de corte no sul do Rio Grande do Sul, agindo como aliados ao enfrentamento de diversas situações de vulnerabilidade, apresentando baixos graus de importância. A ausência ou carência de algumas dessas ferramentas de ação do Estado pode limitar a tomada de decisão dos indivíduos e da família, os quais buscam outras formas de enfrentar ou de se adaptarem às situações de vulnerabilidade.

Ao dedicar atenção ao crédito rural, que figura entre as principais formas de política pública para distintos públicos rurais, constata-se pouco acesso e interesse por parte dos pecuaristas de corte. Portanto, isso nos permite apontar que essa categoria produtiva necessita atualmente de outras formas de políticas públicas, em especial, aquelas que ofereçam acesso à informação para a tomada de decisões e, especialmente, para a comercialização dos seus produtos. 
Por outro lado, o protagonismo das prefeituras municipais mostrase potencial instrumento de enfrentamento e adaptação às situações de vulnerabilidade. Estas, no entanto, não têm se mostrado presentes. Em essência, a medida adotada pelos pecuaristas é não contar com esse ativo, uma vez que é inconstante. Pode-se constatar que organizações públicas governamentais, como Empresa Brasileira de Pesquisa Agropecuária (Embrapa) e Empresa de Assistência Técnica e Extensão Rural do Governo do Rio Grande do Sul (Emater/RS), assumiram um importante papel ao longo do tempo no processo de constituição de autonomia, na medida em que têm prestado suporte na organização social entre pecuaristas e outras organizações. No entanto, esse protagonismo ainda acontece de forma isolada, não consistindo de prática recorrente na organização. A agência de extensão é a que mais preocupa, na medida em que sua atuação junto a esses produtores pouco ou nada aparece nos relatos, e quando presente, envolve a contestação de sua ausência. É necessário entender o que está por trás desse recuo, o qual é reflexo de um engessamento ou sucateamento, mas que tem, sobretudo, gerado situações de vulnerabilidade na pecuária, visto que não são um ativo disponível para situações de risco.

A partir das análises realizadas ao longo do texto, pode-se concluir que a superação dos desafios políticos envolve a constituição de um sistema de governança mais próximo às diferentes realidades rurais, uma vez que as situações de vulnerabilidade podem ser similares entre contextos, mas a disponibilidade de ativos para enfrentá-las varia consideravelmente entre as famílias. Isso exigirá análise, cooperação, coordenação e negociação multidimensional em todas as categorias pecuárias, compreendendo que as diferentes partes interessadas nessa atividade devem desempenhar sua parte como mecanismo para alcançar um consenso e possibilitar os ativos necessários para que os pecuaristas possam reagir às situações de vulnerabilidade. Por isso, fica como reflexão e proposição que ao Estado caberia facultar ações e políticas públicas voltadas à construção de espaços e mecanismos que auxiliem o produtor a tomar decisões, oferecendo o acesso a informações relevantes.

\section{Referências}

ADGER, W. N. Social capital, collective action, and adaptation to climate change. Economic Geography, v. 79, n. 4, p. 387-404, 2003.

ADGER, W. N. Vulnerability. Global Environmental Change, v. 16, n. 3, p. 268281, ago. 2006.

ADGER, W. N. et al. New indicators of vulnerability and adaptive capacity. Norwich: University of East Anglia, 2004. (Technical Report, 7).

ADGER, W. N. et al. Are there social limits to adaptation to climate change? Climatic Change, v. 93, n.3-4, p. 335-354, 2008. 
ANDREATTA, T.; WAQUIL, P. D.; MIGUEL, L. de A. A organização dos estabelecimentos de pecuária de corte de base familiar no Rio Grande do Sul. In: WAQUIL, P. D et al. Pecuária familiar no Rio Grande do Sul: história, diversidade social e dinâmicas de desenvolvimento. Porto Alegre: Editora da UFRGS, 2016. p. 65-86. (Série Estudos Rurais).

BORBA, M. F. S.; TRINDADE, J. P. P. Desafios para conservação e a valorização da pecuária sustentável. In: PILLAR, V. de P.; MÜLLER, S. C.; CASTILHOS, Z. M. de S.; JACQUES, A.V. Á. (Org.). Campos Sulinos - conservação e uso sustentável da biodiversidade. Brasília: Ministério do Meio Ambiente, 2009. p. 391-403.

COMISSÃO ECONÔMICA PARA AMÉRICA LATINA E O CARIBE (CEPAL). Socio-demographic vulnerability: old and new risks for communities, households and individuals. Eletronic Paper. Brasília: UNA, 2002.

CHAMBERS, R. Vulnerability, coping and policy. IDS Bulletin, v. 37, n. 4, Sep. 2006. Disponível em: http://community.eldis.org/.598d23f8. Acesso em: 18 jun. 2011.

CHAMBERS, R.; CONWAY, G. R. Sustainable rural livelihoods: practical concepts for the $21^{\text {st }}$ century. IDS discussion paper. Brighton, n. 296, p.1-33, 1992.

COTRIM, M. S. "Pecuária familiar" na região da Serra do Sudeste do Rio Grande do Sul: um estudo sobre a origem a situação socioagroeconômica do pecuarista familiar no município de Canguçu RS. 2003. Dissertação (Mestrado em Desenvolvimento Rural) - Programa de Pós-Graduação em Desenvolvimento Rural, Universidade Federal do Rio Grande do Sul, Porto Alegre, 2003.

CUTTER, S. L. Vulnerability to environmental hazards. Progress in Human Geography, v. 20, n. 4, p. 529-539, dec. 1996.

DEPONTI, C. M.; SCARTON, L. M.; SCHNEIDER, S. O retrato da extensão rural a partir dos dados do Censo Agropecuário 2006. In: CONGRESSO DA SOCIEDADE BRASILEIRA DE ECONOMIA, ADMINISTRAÇÃO E SOCIOLOGIA RURAL, 50., 2012, Vitória. Anais [...]. Vitória: SOBER, 2012. p. 1-21. 1 CD-ROM.

ELLIS, F. Rural livelihood diversity in developing countries: evidence and policy implications. Natural Resource Perspectives, n. 40, p. 1-10,1999.

ELLIS, F. Rural livelihoods and diversity in developing countries. Oxford: Oxford University Press, 2000.

HERTEL; T. W.; ROSCH, S. D. Climate Change, Agriculture, and Poverty. Applied Economic Perspectives and Policy, n. 5468, p. 5, nov. 2010.

INSTITUTO BRASILEIRO DE GEOGRAFIA E ESTATÍSTICA. Censo Agropecuário 2006. Rio de Janeiro: IBGE, 2009.

JANSSEN, M.A.; OSTROM, E. Resilience, vulnerability, and adaptation: a crosscutting theme of the International Human Dimensions Programme on Global Environmental Change. Global Environmental Change, n. 16, n. 3, p. 237-239, aug. 2006.

$\mathrm{KIRSCH}, \mathrm{H}$. M. Vulnerabilidade e resiliência social da agricultura familiar do Alto Guaporé (MT) em contexto de mudanças ambientais contemporâneas. 
2015. 333 f. Tese (Doutorado em Desenvolvimento Rural) - Programa de PósGraduação em Desenvolvimento Rural, Universidade Federal do Rio Grande do Sul, Porto Alegre, 2015.

KIRSCH, H. M. et al. A agricultura em um cenário de mudanças ambientais: a importância das formas de manejo para a sustentabilidade. Espacios (Caracas), v. 37, p. 3, 2016.

LITRE, G. Os gaúchos e a globalização: vulnerabilidade e adaptação da pecuária familiar no pampa do Uruguai, Argentina e Brasil. 2010. 474 f. Tese (Doutorado em Desenvolvimento Sustentável e Géographie et Aménagement du Territoire) Universidade de Brasília, Université Paris III - Sorbonne Nouvelle, Brasília, 2010.

MARANDOLA JR., E.; HOGAN, D.J. Vulnerabilidades e riscos: entre geografia e demografia. Revista Brasileira de Estudos da População, São Paulo, v. 22, n. 1, p. 29-53, jan./jun. 2005.

MARANDOLA JR., E.; HOGAN, D. J. As dimensões da vulnerabilidade. São Paulo em Perspectiva, São Paulo, v. 20, n. 1, p. 33-43, jan./mar. 2006.

MATTE, A. Vulnerabilidade, capacitações e meios de vida dos pecuaristas de corte da Campanha Meridional e Serra do Sudeste do Rio Grande do Sul. 2013. Dissertação (Mestrado em Desenvolvimento Rural) - Programa de PósGraduação em Desenvolvimento Rural, Universidade Federal do Rio Grande do Sul, Porto Alegre, 2013.

MATTE, A.; WAQUIL, P. D. Vulnerabilidade, capacitações e meios de vida de pecuaristas de corte no sul do Rio Grande do Sul. In: WAQUIL, P. D. et al. Pecuária familiar no Rio Grande do Sul: história, diversidade social e dinâmicas de desenvolvimento. Porto Alegre: Editora da UFRGS, 2016. p. 237-264. (Série Estudos Rurais).

MATTE, A.; WAQUIL, P. D. Vulnerabilidade social e a construção de estratégias de enfrentamento e adaptação para pecuaristas de corte no Rio Grande do Sul. Desenvolvimento e Meio Ambiente, Curitiba (UFPR), v. 28, p. 107-125, jul./ dez. 2013.

MATTE, A. et al. Mercado de cadeias curtas na pecuária familiar: um processo de relocalização no território Alto Camaquã no Sul do Rio Grande do Sul/Brasil. Redes, Edição Especial Agricultura Familiar: processos e práticas, Santa Cruz do Sul, v. 21, n. 3, p. 137-158, set./dez. 2016.

MORTON, J. F. The impact of climate change on smallholder and subsistence agriculture. PNAS, v. 104, n. 50, p. 19680-19685, dec. 2007.

NOBRE, C. A. Mudanças Climáticas e o Brasil - Contextualização. In: Parcerias Estratégicas, n. 27. Mudanças do clima no Brasil: vulnerabilidade, impactos e adaptações, Brasília: Centro de Gestão e Estudos Estratégicos, Ministério da Ciência e Tecnologia, 2008.

RIBEIRO, C. M. O modo de vida dos pecuaristas familiares no pampa brasileiro. In: WAQUIL, P. D. et al. Pecuária familiar no Rio Grande do Sul: história, diversidade social e dinâmicas de desenvolvimento. Porto Alegre: Editora da UFRGS, 2016. p. 87-108. (Série Estudos Rurais).

RIBOT, J. C. Vulnerability does not just fall from the sky: toward multi-scale propoor climate policy. In: MEARNS, R.; NORTON, A. (Ed.). Social dimensions 
of climate change: equity and vulnerability in a warming world. Washington, DC: The World Bank, 2009.

RICHARDSON, R. J. Pesquisa Social: métodos e técnicas. 3.ed. revista e ampliada, São Paulo: Atlas, 2009.

SABOURIN, E. Camponeses do Brasil: entre a troca mercantil e a reciprocidade. Rio de Janeiro: Ed. Garamond, 2009.

SANDRINI, G. B. D. Processo de inserção dos pecuaristas familiares do Rio Grande do Sul na cadeia produtiva da carne. 2005. Dissertação (Mestrado em Desenvolvimento Rural) - Programa de Pós-Graduação em Desenvolvimento Rural, Universidade Federal do Rio Grande do Sul, Porto Alegre, 2005.

SCOONES, I. Livelihoods perspectives and rural development. Journal of Peasant Studies, v. 36, n. 1, p. 171-196, jan. 2009.

TOIGO, C. H.; CONTERATO, M. A. Pobreza, Vulnerabilidade e Desenvolvimento no Território Rural Zona Sul: o que aponta o Índice de Condição de Vida?. RESR, Piracicaba, v. 55, n. 2, p. 267-284, abr./jun. 2017.

Submetido em: 06/08/2018

Aceito em: 10/10/2018 


\title{
SITUATIONS OF VULNERABILITY IN BEEF CATTLE IN RIO GRANDE DO SUL: BETWEEN PUBLIC POLICIES AND CURRENT RURAL ISSUES
}

\begin{abstract}
The objective of this article was to understand the degree of vulnerability on different rural issues related to the State action in relation to the livelihoods of cattle farmers in southern Rio Grande do Sul, analyzing how these producers create coping strategies or adaptation to the effects of the situations of vulnerability to which they are exposed. The livelihoods approach is used as an analytical tool to study situations of vulnerability. The empirical data comes from 60 interviews with cattle ranchers from the municipalities of Bagé, Dom Pedrito, Pinheiro Machado and Piratini, south of Rio Grande do Sul. The results indicate that among the seven vulnerability factors analyzed, the presence of the State, through the actions of the municipal government and agencies of extension and research, it is important to reactive to situations of vulnerability when accessible to the rural producer. Livestock farmers seek local organizations such as trade unions and producer associations, mechanisms to respond to vulnerability, as well as the mobilization of domestic assets to rural property, in the fragility of services that should be offered by public policies. Overcoming political challenges involves building governance systems closer to the different rural realities, facilitated by understanding the situations of vulnerability that can be generated by the presence or absence of the state.
\end{abstract}

Keywords: Means of life. Beef cattle. Public policy. Vulnerability.

\section{SITUACIONES DE VULNERABILIDAD EN LA GANADERÍA DE CORTE EN RIO GRANDE DO SUL: ENTRE POLÍTICAS PÚBLICAS Y CUESTIONES RURALES ACTUALES}

\begin{abstract}
Resumen
El objetivo del artículo fue comprender el grado de vulnerabilidad sobre diferentes cuestiones rurales vinculadas a la acción del Estado en relación a los medios de vida de ganaderos de corte del sur de Rio Grande do Sul, analizando cómo esos productores crean estrategias de enfrentamiento o de adaptación a los efectos de las situaciones de vulnerabilidad a las que están expuestos. El enfoque de los medios de vida se utiliza como instrumental analítico para estudiar situaciones de vulnerabilidad. Los datos empíricos provienen de la realización de 60 entrevistas con ganaderos de corte de los municipios de Bagé, Don Pedrito, Pinheiro Machado y Piratini, al sur de Rio Grande do Sul. Los resultados apuntan que entre los siete factores de vulnerabilidad analizados, la presencia del Estado, por medio de la actuación de la alcaldía municipal y de agencias de extensión e investigación, es importante activo para la reacción a las situaciones de vulnerabilidad cuando es accesible al productor rural. Los ganaderos buscan en organizaciones locales, como sindicatos y asociaciones de productores, mecanismos para reaccionar ante la vulnerabilidad, así como la movilización de activos internos a la propiedad rural, cuando la fragilidad de la atención de servicios que deberían ser ofrecidos por políticas públicas. La superación de los desafíos políticos implica la constitución de sistemas de gobernanza más cercanos a las diferentes realidades rurales, facilitada cuando se comprenden las situaciones de vulnerabilidad que pueden ser generadas por la presencia o ausencia del Estado.
\end{abstract}

Palabras clave: Medios de vida. Pecuaria de corte. Políticas públicas. Vulnerabilidad. 